\title{
Numerical Study of Water Flooding Simulations Using ANSYS Fluent
}

\author{
Mohammed Azhar ${ }^{1}$, Jay Sanyal² \\ ${ }^{1}$ ANSYS UK Ltd. \\ 6 Europa View, Sheffield S9 1XH, UK \\ mohammed.azhar@ansys.com,jay.sanyal@ansys.com \\ ${ }^{2}$ ANSYS Inc. \\ 10 Cavendish Court, Lebanon, New Hampshire 03766-1442, USA
}

\begin{abstract}
Reservoir flow simulations are frequently used for predicting reservoir performances. Often time many petrochemical properties are required for making these predictions. But, two parameters that have the most significant impact on the performance prediction are relative permeability and capillary pressure curves. These data are usually calculated from laboratory measurements using reservoir core samples and reservoir fluids. The main drawback of this approach is that the calculated values are subject to systematic errors such as the end effects. The accuracy of these data can be improved by using numerical models.

Over the past decade, computational fluid dynamics has received considerable attention for simulating two-phase flow in porous media. This tool can be used to construct core models and make laboratory results more reliable and accurate. In this paper, we attempt to analyse laboratory experiments in ANSYS Fluent software. A quantitative demonstration is performed for typical relative permeabilities and reservoir conditions. The numerical simulations developed in this paper show good comparison with analytical and special core analysis simulator solutions.
\end{abstract}

Keywords: ANSYS Fluent, computational fluid dynamics, immiscible displacement, oil, porous media, reservoir flow, special core analysis Simulator, two-phase flow, water

\section{Introduction}

Reservoir flow simulations are used by petroleum companies to help in the development of new fields and in developed areas where production forecasts are needed. The quality of reservoir predictions depends on several factors, such as raw data measurements, and petrophysical modelling. The most critical factor is an accurate and detailed relative permeability and capillary pressure data. Nevertheless, reservoir simulation is regularly used with great success; and even if there may be uncertainties tied to simulated results, the simulations reveal invaluable information about the reservoir flow. Commercial reservoir simulators such as Eclipse can predict the possible production rates and overall production resulting from production strategies. Standard large-scale reservoir simulators, however, typically do not consider basic near-well physics such as well completion components and details around the wellbore and therefore are not able to provide good results.

Commercial CFD simulators, on the other hand, have several models implemented in their solvers for a variety of situations. Along with the versatility of geometry and mesh creation, the CFD simulators can be used with great potential for reservoir simulation. ANSYS Fluent CFD software has a built-in two-phase flow porous media model suitable for petroleum reservoir flows. The two-phase flow porous media model is based on the extended Darcy-Forchheimer-Brinkman model coupled with the Corey-Brookes relative permeability model. This model is standard and well known in commercial software for reservoir simulations.

\section{Benchmark validation}

To evaluate the two-phase flow porous media model in ANSYS Fluent several problems relevant to petroleum engineering applications are presented. In all cases, we consider two-phase infiltration of water into porous media initially filled oil and connate water. The first example considers the classic 1d Buckley-Leverett problem in homogenous medium with different fluid properties and zero capillary pressure. In example 2, we compare the numerical solution for core flooding problem with Special Core Analysis (SCORES) [7] solution. In the last example for the robustness the numerical solution in a $3 \mathrm{~d}$ reservoir model. 
It worth mentioning here that the two-phase porous media model in ANSYS Fluent is based on extended DarcyForchheimer model. The precise form of the source term used in the momentum equations is as follows:

$$
\text { source }=-\left(\alpha_{q}^{2} \gamma^{2} \frac{\mu_{q} V_{q}}{K k_{r, q}}+\alpha_{q}^{3} \gamma^{3} \frac{C_{2} \rho_{q}\left|V_{q}\right| V_{q}}{2}\right)
$$

Where $\alpha_{\mathrm{q}}$ is the phase volume fraction, $\gamma$ is the rock porosity, $\mu_{\mathrm{q}}$ is phase laminar viscosity, $K$ is the absolute permeability, and $\mathrm{k}_{r \mathrm{q}}$ is phase relative permeability, $\mathrm{C}_{2}$ is a constant and $\rho_{\mathrm{q}}$ is the phase density.

To evaluate the two-phase flow porous media model in ANSYS Fluent several problems relevant to petroleum engineering applications are presented. In all cases, we consider two-phase infiltration of water into porous media initially

The parameter relative permeability is a scalar that quantifies how one phase flows relative to the other. In the oil and gas industry, it is typically calculated using empirical correlation such as Corey model [1]. According to Corey the phase relative permeability varies with saturation (phase volume fraction) and has the general form:

$$
k_{r, q}=k_{r, q}^{0} S_{q}^{n q}
$$

Where the parameter $S_{q}$ is the normalised saturation of the $q^{t h}$ phase. $K_{r, q}^{o}$ is the maximum or the endpoint phase relative permeability and the parameter $n q$ is the $q^{\text {th }}$ phase Corey exponent. For reservoir modelling the water and oil relative permeabilities in two-phase flows are as follows:

$$
k_{r, w}=k_{r, w}^{0} \alpha_{w}^{n w} \quad k_{r, o}=k_{r, 0}^{0}\left(1-\alpha_{o}\right)^{n 0}
$$

Where the $\alpha_{w}$ and $\alpha_{o}$ are the residual saturation.

\subsection{Example}

\subsubsection{Buckley-Leverett problem}

We first verify our CFD model with known analytical solutions in 1d space. We consider a horizontal homogenous domain of length $1 \mathrm{~m}$ and height $0.05 \mathrm{~m}$, initially saturated with oil. Water is injected at a constant velocity of $5 . e-5 \mathrm{~m} / \mathrm{s}$ is injected at one end to displace oil at the other end. In all cases, the pressure is kept constant, and the capillary pressure is neglected. Table 1 provides the relevant data for this problem. We use the conventional pressure-based solver to numerically solve this problem in ANSYS Fluent and compare the results to the analytical solutions. 
Table 1: Fluid and rock properties for the Leverett-Buckley Simulation.

\begin{tabular}{|l|c|c|}
\hline Property & Value & Unit \\
\hline Water density, $\boldsymbol{\rho}_{\boldsymbol{w}}$ & 1000 & $\mathrm{~kg} / \mathrm{m}^{3}$ \\
\hline Oil density, $\boldsymbol{\rho}_{\boldsymbol{o}}$ & 800 & $\mathrm{~kg} / \mathrm{m}^{3}$ \\
\hline Water viscosity, $\boldsymbol{\mu}_{\boldsymbol{w}}$ & 1 & $\mathrm{cP}$ \\
\hline Oil viscosity, $\boldsymbol{\mu}_{\boldsymbol{w}}$ & 10 & $\mathrm{cP}$ \\
\hline Rock porosity, $\boldsymbol{\gamma}$ & 0.2 & - \\
\hline Absolute rock permeability, $\boldsymbol{K}$ & $4.739 \mathrm{e}-9$ & $\mathrm{mD}$ \\
\hline Residual water saturation, $\boldsymbol{\alpha}_{\boldsymbol{w}}$ & 0 & - \\
\hline Residual oil saturation, $\boldsymbol{\alpha}_{\boldsymbol{o}}$ & 0 & - \\
\hline Endpoint relative permeability of water, $\boldsymbol{k}_{\boldsymbol{r}, \boldsymbol{w}}^{\mathbf{n}}$ & 1 & - \\
\hline Endpoint relative permeability of oil, $\boldsymbol{k}_{\boldsymbol{r}, \boldsymbol{o}}$ & 1 & - \\
\hline Corey exponent water, $\boldsymbol{n} \boldsymbol{w}$ & 1 & - \\
\hline Corey exponent oil, $\boldsymbol{n o}$ & 1 & \\
\hline
\end{tabular}

In this example, we assume different viscosity for oil and water phases and use linear relative permeability. Three different mesh resolutions with 500, 2000 and 8000 cells are used for numerical simulations. To avoid/minimise any influence from the symmetry boundary conditions, we resolved the mesh in the vertical or the cross-sectional direction. Figure 1 shows the geometry and mesh for the coarse mesh resolution.

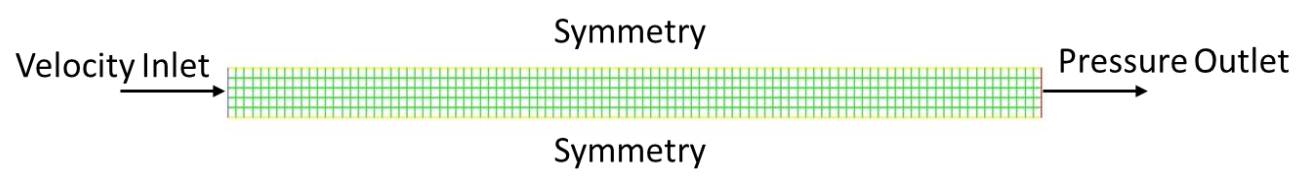

Fig. 1: Geometry and mesh for the Buckley-Leverett Problem.

In Figure 2 the numerical solutions for the water volume fraction at time of $4657.5 \mathrm{~s}$ in displacement process for the three different mesh resolutions are plotted. The waterfront position and height are accurately captured with relatively low numerical dispersion. Furthermore, the numerical results are virtually identical for all three mesh resolutions.

In Figure 3 we compare numerical results for a three different time step sizes corresponding to Courant numbers of 1 , 0.1 and 0.01 . The numerical solutions for Courant numbers of 0.1 and 0.001 are virtually identical, indicating timeindependent solutions. The results also suggest that the time step size based on the Courant number of 1 is too large for predicting an accurate solution. Therefore, considering both computational accuracy and efficiency, time step based on Courant number of 0.1 gives a good approximation for the solution. It is worth mentioning at this conjuncture that in all cases we used the default multiphase solver settings.

In Figure 4 we plot the numerical results for the two different time steps and compare results with the Buckley-Leverett or the frontal analysis solution [2]. We use a linear approximation for the relative permeabilities. The comparison shows a good agreement between the analytical and numerical solutions.

\subsection{Example}

\subsubsection{Core flooding}

We next validate the CFD model against SCORES, a web-based interface to Shell's propriety SCAL simulator. SCORES has been validated against three different industry standard simulators used for determining the relative permeability and capillary pressure data from laboratory experiments. 
We consider a two-phase flow in a $2 \mathrm{~d}$ horizontal axisymmetric domain of homogeneous medium of $8 \mathrm{~cm}$ length and $4 \mathrm{~cm}$ diameter with a porosity of 0.2 and isotropic permeability of $326 \mathrm{mD}$. The medium is initially saturated with residual water saturation, and the rest is filled with oil. Water is injected uniformly at a constant velocity of $1 . e-5 \mathrm{~m} / \mathrm{s}$ from one end to displace oil to the other end. The pressure is kept constant and capillary pressure is neglected. The relevant parameters for this problem are provided in Table 2.

In Figure 5 we compare the calculated water phase average volume fraction with SCORES predictions. The initial slope of the predicted saturation curve is in a good agreement with SCORES. However, the numerical simulation significantly overestimates the saturation curve plateau. It seems that the injected water flows slowly in the numerical simulations. The process by which water enters a cell and increases the relative water permeability is more gradual resulting in a delayed water breakthrough.
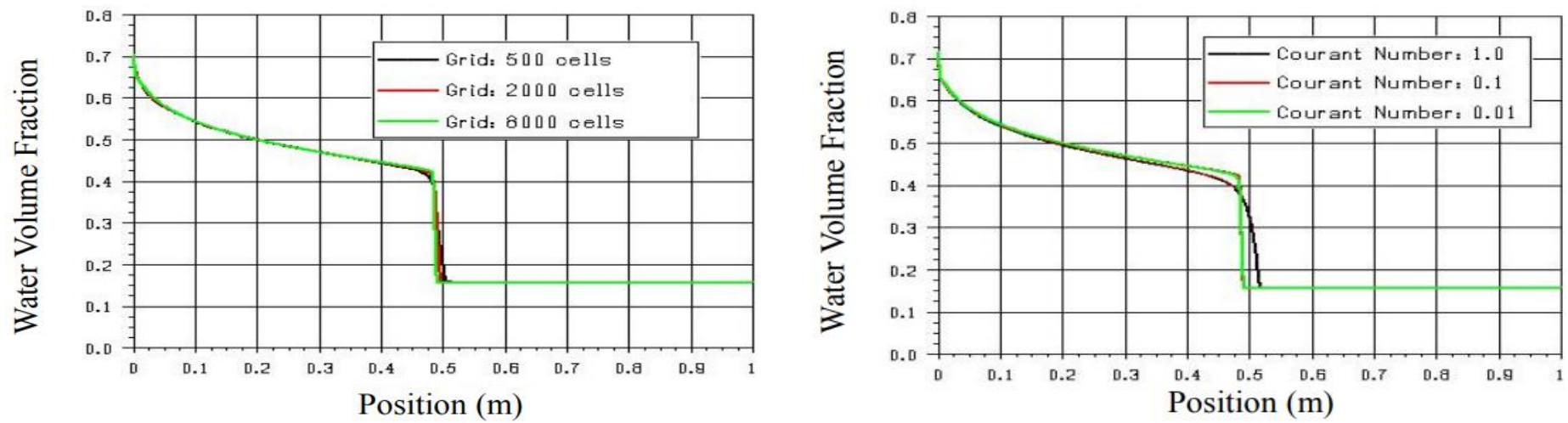

Water saturation profiles during 4657.5 seconds of water flooding

Fig. 2: Examination of grid dependence.

Fig. 3: Examination of the time dependence.

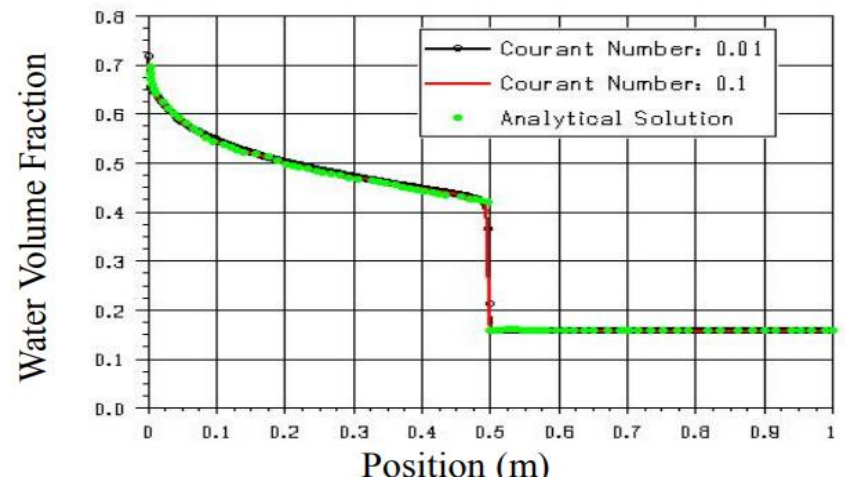

Fig. 4: Water saturation profile. 
Table 2: Fluid and rock properties used for Scores Simulation.

\begin{tabular}{|l|c|c|}
\hline Property & Value & Unit \\
\hline Water density, $\boldsymbol{\rho}_{\boldsymbol{w}}$ & 1000 & $\mathrm{~kg} / \mathrm{m}^{3}$ \\
\hline Oil density, $\boldsymbol{\rho}_{\boldsymbol{o}}$ & 800 & $\mathrm{~kg} / \mathrm{m}^{3}$ \\
\hline Water viscosity, $\boldsymbol{\mu}_{\boldsymbol{w}}$ & 1 & $\mathrm{cP}$ \\
\hline Oil viscosity, $\boldsymbol{\mu}_{\boldsymbol{w}}$ & 1 & $\mathrm{cP}$ \\
\hline Rock porosity, $\boldsymbol{\gamma}$ & 0.25 & - \\
\hline Absolute rock permeability, $\boldsymbol{K}$ & 100 & $\mathrm{mD}$ \\
\hline Residual water saturation, $\boldsymbol{\alpha}_{\boldsymbol{w}}$ & 0.2 & - \\
\hline Residual oil saturation, $\boldsymbol{\alpha}_{\boldsymbol{o}}$ & 0.2 & - \\
\hline Endpoint relative permeability water, $\boldsymbol{k}_{\boldsymbol{r}, \boldsymbol{w}}^{\mathbf{n}}$ & 0.5 & - \\
\hline Endpoint relative permeability oil, $\boldsymbol{k}_{\boldsymbol{r}, \boldsymbol{o}}^{\mathbf{0}}$ & 0.5 & - \\
\hline Corey exponent water, $\boldsymbol{n} \boldsymbol{w}$ & 3 & - \\
\hline Corey exponent oil, $\boldsymbol{n o}$ & 3 & - \\
\hline
\end{tabular}

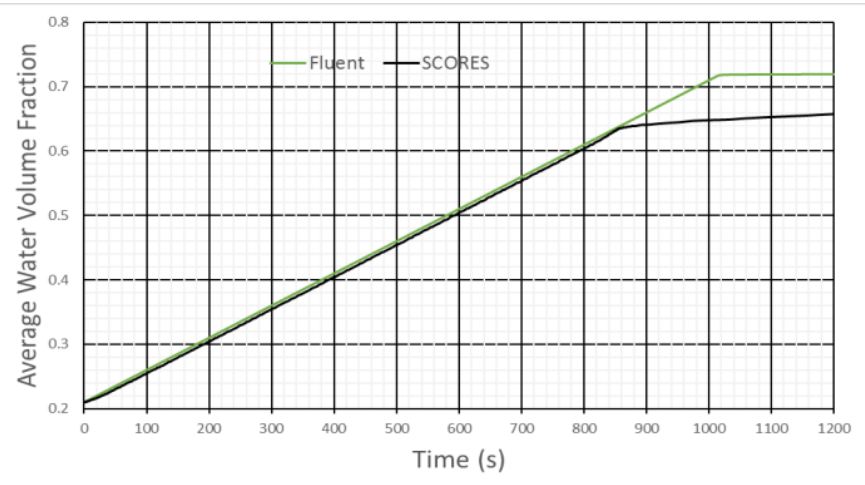

Fig. 5: Average water saturation curve as a function of time.

\subsection{Example}

\subsubsection{3d reservoir flow problem}

The purpose of this last example is to show the robustness of the ANSYS Fluent Solver in modelling a practical reservoir flow problem. We consider a 3d homogeneous anisotropic medium with dimensions (762 m x $762 \mathrm{~m} \times 152.4 \mathrm{~m}$ ). The injection and production wells are located $(0 \mathrm{~m}, 0 \mathrm{~m}, 0 \mathrm{~m})$ and $(762 \mathrm{~m}, 762 \mathrm{~m}, 152.4 \mathrm{~m})$, respectively. The injection rate is $3.86 \mathrm{~m}^{3} / \mathrm{s}$. The rock and fluid properties are provided in Table 3 . We consider a single mesh and discretised the domain into 68600 hex cells. The geometry and the computational mesh for the reservoir flow problem is shown in Figure 6.

In Figure 7 we display the contour of oil saturation at four different times. The results show the flow around the injector well is radial. During the first 250 hours of flooding, injected water sweeps the oil away from the injection well towards the production well. The oil displacement is higher along the lower end of the reservoir due to the higher permeability in a horizontal plane and the effect of gravity. However, after the front reaches corner diagonally opposite the injector well, the no-flow condition along the border alters the radial flow pattern. The incompressible nature of fluids causes the flow field in the corner to transition from radial to quasi-radial. At 750 hours, we see the waterfront flows faster near the producer well. Consequently, oil saturation remains high around the corner region directly opposite the injector well. As the physical time approaches 1000 hours and 1500 hours, we see that the maximum oil saturations decrease to 0.67 and 0.57 , respectively. 
Table 3: Fluid and rock properties used for 3d reservoir model.

\begin{tabular}{|l|c|c|}
\hline Property & Value & Unit \\
\hline Water density, $\boldsymbol{\rho}_{\boldsymbol{w}}$ & 958 & $\mathrm{~kg} / \mathrm{m}^{3}$ \\
\hline Oil density, $\boldsymbol{\rho}_{\boldsymbol{o}}$ & 639 & $\mathrm{~kg} / \mathrm{m}^{3}$ \\
\hline Water viscosity, $\boldsymbol{\mu}_{\boldsymbol{w}}$ & 0.31 & $\mathrm{cP}$ \\
\hline Oil viscosity, $\boldsymbol{\mu}_{\boldsymbol{w}}$ & 0.91 & $\mathrm{cP}$ \\
\hline Rock porosity, $\boldsymbol{\gamma}$ & 0.3 & - \\
\hline Absolute rock permeability, $\boldsymbol{K}$ & 200 & $\mathrm{mD}$ \\
\hline Residual water saturation, $\boldsymbol{\alpha}_{\boldsymbol{w}}$ & 0.2 & - \\
\hline Residual oil saturation, $\boldsymbol{\alpha}_{\boldsymbol{o}}$ & 0.25 & - \\
\hline Endpoint relative permeability water, $\boldsymbol{k}_{\boldsymbol{r}, \boldsymbol{w}}^{\mathbf{0}}$ & 0.3 & - \\
\hline Endpoint relative permeability oil, $\boldsymbol{k}_{\boldsymbol{r}, \boldsymbol{o}}^{\mathbf{o}}$ & 0.68 & - \\
\hline Corey exponent water, $\boldsymbol{n} \boldsymbol{w}$ & 3 & - \\
\hline Corey exponent oil, $\boldsymbol{n o}$ & 3 & - \\
\hline
\end{tabular}

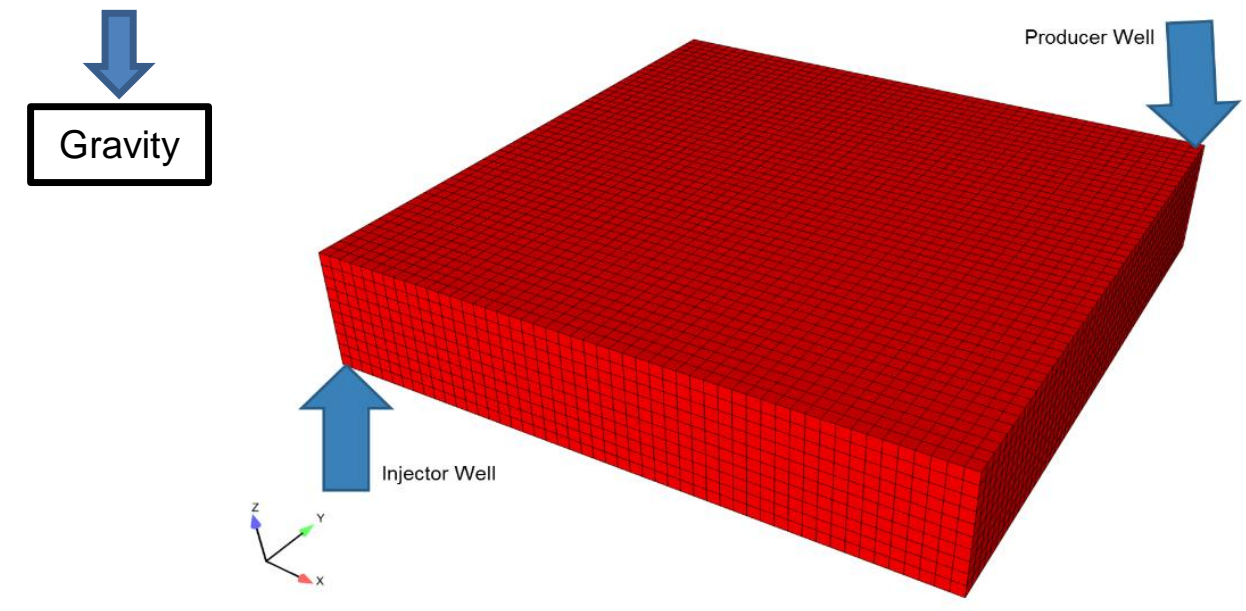

Fig. 6: Geometry and mesh for the reservoir flow problem. 


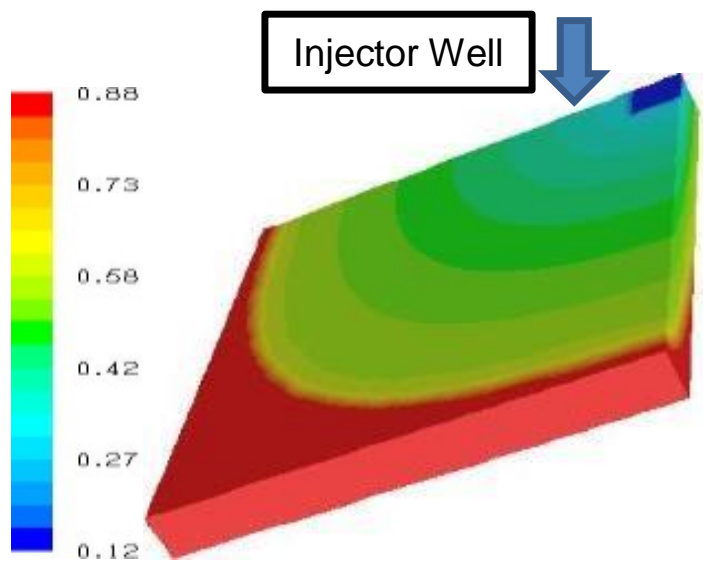

Time $=250$ hours

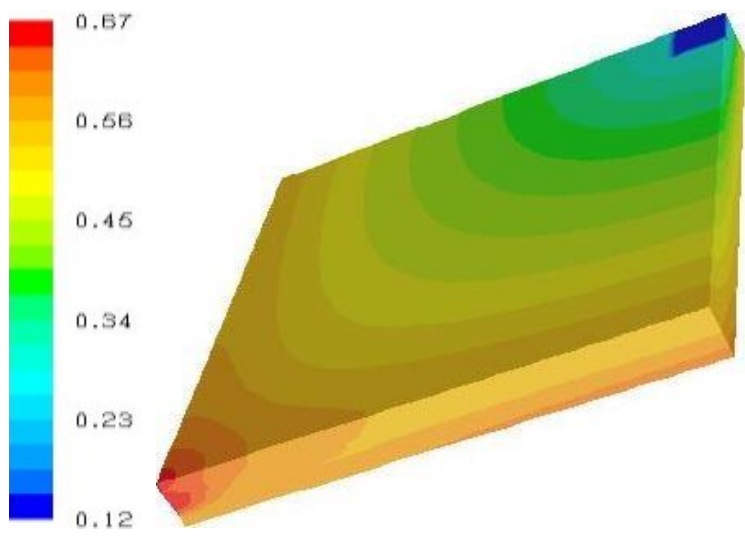

Time $=1000$ hours

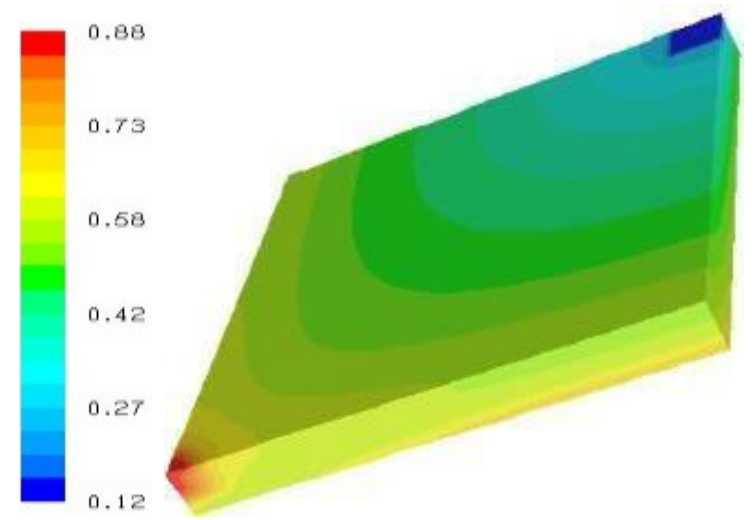

Time $=750$ hours

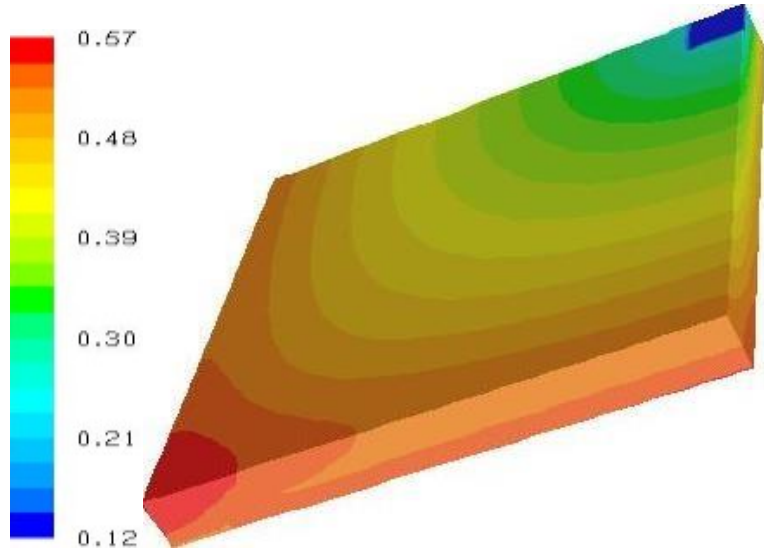

Time $=1500$ hours

Fig. 7: Water saturation at various times during water flooding.

\section{Conclusions}

The multiphase porous media model has been successfully used to simulate transient oil-water flows in lab and reservoir flow conditions. The 1D analysis has correctly reproduced the Buckley-Leverett analytical solution with linear Corey correlation. The numerical solutions are time-step and grid independent. The $2 \mathrm{~d}$ and $3 \mathrm{~d}$ simulations have demonstrated that the present model can successfully capture the flow characteristics and ensure a physically bounded solution. The multiphase solver is fast and robust allowing time steps to be as large as few hours for a reservoir with the real flow time of 2-20 years, showing a great promise for practical reservoir flow performance analysis.

\section{Acknowledgements}

The authors would like to thank Dias, R.A.C., and Oliveira Jr., J.A.A at ESSS of sharing their deep knowledge of reservoir/well analyses and petroleum exploring. The authors appreciate greatly for them to provide us with the validation cases, porous properties and meaningful flow conditions for reservoir modelling. 


\section{List of Symbols \\ Greek Variables}

$\alpha_{\mathrm{q}}$

$\gamma$

$\mu_{\mathrm{q}}$

$\rho_{\mathrm{q}}$

\section{Latin Variables}

$\mathrm{C}_{2}$

$n q$

K

$k_{r, q}$

$K_{r, q}^{o}$

$S_{q}$ is the normalised saturation
Phase volume fraction [-]

Rock porosity [-]

Dynamic viscosity of phase $\mathrm{q}[\mathrm{P}]$

Density of phase $\mathrm{q}\left[\mathrm{kg} \mathrm{m}^{-3}\right]$

Inertial loss constant [-]

the $q^{\text {th }}$ phase Corey exponent

Intrinsic permeability tensor $\left[\mathrm{m}^{2}\right]$

Relative permeability of phase q [-]

Endpoint relative permeability of phase q [-]

Normalised saturation of phase q [-]

\section{References}

[1] R. Brooks and Corey," Hydraulic properties of porous media", Tech. rep., Hydrology Paper 3, Colorado State University, Fort Collins, Colorado, USA, 1964.

[2] S. E. Buckley, and M. C. Leverett, "Mechanism of Fluids Displacement in Sands," Trans. AIME, vol. 146, pp. 107$116,1942$.

[3] H. Li, and S. A.Vasquez, "A General Purpose Physical Velocity Formulation for Numerical Simulation of Flows through Porous Media", Proceedings of the 2nd International Conference on Porous Media and its Application in Science and Engineering, ICPM2, 2007, Kauai, Hawaii, USA.

[4] R. A. C. Dias, and J. A. A. Oliveira Jr., "Multiphase Flow in Porous Media - FLUENT," ESSS Technical Presentation, 2010.

[5] S. A. Vasquez, "Multiphase in Porous Media with ANSYS FLUENT: Buckley-Leverett Analysis," ANSYS Internal Technical Note, 2011.

[6] Multiphase Flows, Theory Guide, ANSYS FLUENT 17.0 Documentation, 2015.

[7] J. G. Mass, "Computer simulation of Special Core Analysis (SCAL) flow experiments shared on the Internet" SCA-9719. 